\title{
Perfil Epidemiológico da Violência Contra Crianças e Adolescentes no Estado de Minas Gerais, Brasil
}

\section{Epidemiological Profile of Violence against Children and Adolescents in the State of Minas Gerais, Brazil}

Sérgio Ricardo Del Bel Antognollia; Gabriela Fernandes de Oliveiraa; Breno Resende Rodrigues da Cunhaa; Paula Monikee Rezende Alves ${ }^{a}$; Leonardo dos Reis Duarte Silva ${ }^{a}$; Mariana Giorgiania ${ }^{a}$ Juliana Rizza Ribeiro Batista ${ }^{a}$; Anderson de Cintra Souza ${ }^{\mathrm{a}}$; Ana Clara Ferraz Sousa ${ }^{\mathrm{a}}$; Henrique Cardoso Marcene ${ }^{\mathrm{a}}$; Stefan Vilges de Oliveira*a

\begin{abstract}
Resumo
Diante de poucos estudos referentes à epidemiologia dos casos de violência contra crianças e adolescentes praticados no país, com resultados ainda menores ao se restringir ao Estado de Minas Gerais se faz necessário explorar esse fenômeno e suas características. O presente estudo tem como objetivo analisar o perfil epidemiológico dos casos de violência praticados contra a população infantil e juvenil do Estado de Minas Gerais, entre os anos de 2007 e 2017. A metodologia utilizada foi um estudo descritivo, a partir de dados secundários obtidos do Sistema de Informação de Agravos de Notificação (SINAN). A partir da análise de dados, foram registradas 693.495 notificações de casos de violência contra crianças e adolescentes no período relatado, sendo que $3.600(0,51 \%)$ desses evoluíram para óbito. As vítimas foram, predominantemente, do sexo feminino com 421.715 (60,81\%), negras $304.296(43,87 \%)$, na faixa etária de 15 a 19 anos com $256.612(37,0 \%)$, com baixa escolaridade 196.914 (28,4\%). Foi observado o predomínio da violência física em 332.369 (47,93\%), praticada por familiar em 268.857 (38,76\%), com relato de uso de álcool em 98.563 (14,21\%). Assim, este estudo realça a necessidade de maior atenção a essa população vulnerável e de estabelecimento de ações efetivas no combate a esse tipo de violência.
\end{abstract}

Palavras-chave: Brasil. Epidemiologia. Violência. Criança. Adolescente. Vigilância em Saúde.

\begin{abstract}
Considering the small number of studies found regarding the violence epidemiology against children and adolescents practiced in the country, with even smaller results when restricting the search to the state of Minas Gerais $(M G)$, it proved necessary to explore this phenomenon and its characteristics. The present study aims to analyze the violence epidemiological profile against the child and youth occurrences in MG, between the years 2007 and 2017. The methodology used was a descriptive study, based on secondary data obtained from the Notifiable Diseases Information System (SINAN). Taking the data analysis into account, 693,495 notifications of violence against children and adolescents occurrences were recorded in the reported period, of which 3,600 (0.51\%) led to death. The victims were predominantly female, representing 421,715 (60.81\%) of them, 304,296 (43.87\%) were black, 256,612 (37.0\%) were aged between 15 and 19 years old and 196,914 (28.4\%) had low education. There was a predominance of physical violence in 332,369 (47.93\%) of the cases, 268,857 (38.76\%) were practiced by a relative, with reports of alcohol use in 98,563 (14.21\%) occurrences. Thus, this study highlights the urge for greater attention to this vulnerable population and for the establishment of effective actions to combat this sort of violence.
\end{abstract}

Keywords: Brazil. Epidemiology. Violence. Children. Adolescent. Surveillance in Health.

\section{Introdução}

Para entender o perfil epidemiológico da violência contra a criança, é preciso definir os principais termos envolvidos. De acordo com a Organização Mundial da Saúde (OMS), "criança" é um indivíduo com até 19 anos, a menos que a lei nacional defina uma idade adulta precoce (WHO, 2013). No caso do Brasil, o Estatuto da Criança e do Adolescente (ECA) define "criança" como indivíduo de até 12 anos incompletos e "adolescente" entre 12 e 18 anos (BRASIL, 1990, p.1). No entanto, para aplicações de determinadas leis, o ECA se estende às pessoas entre dezoito e vinte e um anos de idade. Dessa forma, a definição da OMS será utilizada na abordagem da violência, pois é mais abrangente quanto à faixa etária e não possui variações de acordo com a lei a que se aplica, além de propiciar a comparação do cenário encontrado com dados mundiais.

Ademais, a OMS também define o termo "violência" em relação a essa faixa etária, sendo qualquer forma de maus tratos físicos e emocionais, abuso sexual e atos de negligência contra crianças, assim como a sua exploração comercial e com outros fins, que resulte em dano real ou potencial à saúde, à sobrevivência e ao desenvolvimento da criança no contexto de uma relação de responsabilidade, confiança ou poder (WHO, 2016).

Acerca das modalidades, a violência intrafamiliar se manifesta de quatro formas principais: física, sexual, psicológica e negligência. Normalmente, são praticadas por pais, responsáveis e/ou pessoas da confiança de crianças e adolescentes. É importante destacar que nem sempre as manifestações de violência vão deixar marcas físicas, mas que 
nem por isso deixam de ser graves em função das consequências emocionais, marcas psíquicas e afetivas que existirão (REIS $\mathrm{DM}$ et al, 2018). Ainda, no cenário intrafamiliar, vale citar que também se considera violência a omissão da ação por parte de algum integrante da família, ainda que sem laços sanguíneos, cujo desfecho gere qualquer dano àquele que sofreu a ação (MAGALHÃES JRF et al, 2017).

Dentro do contexto brasileiro, quaisquer tipos de violência contra crianças e adolescentes são reconhecidos como um problema de saúde pública, pois suas implicações podem se estender desde gastos em programas de proteção e sistema prisional até prejuízos nas esferas da saúde e qualidade de vida, além de óbito. Portanto, cabe ao legislativo brasileiro promover estratégias de proteção à criança e ao adolescente. Nesse sentido, foi estabelecido no Art. 13 do ECA, que notificações de suspeitas ou confirmações da violência são compulsórias e devem ser reportadas ao Conselho Tutelar (MACEDO et al., 2019).

Apesar desde ser o órgão de referência para acolhimento dos casos e encaminhamento das vítimas e familiares, a notificação também pode ser realizada junto a outras instituições, que atuem de forma articulada, como as Delegacias de Proteção da Criança e do Adolescente e o Ministério Público (MACEDO et al., 2019).

No entanto, em 53 municípios de Minas Gerais, no período de 2013 a 2015, verificou-se que há poucas notificações de violências no Sistema de Informações de Agravos de Notificação (SINAN), mesmo após o Estado já ter adotado a obrigatoriedade de sua notificação até mesmo nos casos de suspeita (SOUTO, 2018).

Entre os anos de 2010 e 2014, foram aprovados: Proposta de Emenda Constitucional da Juventude, o Plano Nacional de Enfrentamento da Violência Sexual contra Crianças e Adolescentes, a Lei $\mathrm{n}^{\mathrm{o}} 12.852$, que instituiu o Estatuto de Juventude, e a Lei $\mathrm{n}^{\circ} 13.010$, também conhecida como Lei da Palmada. Essas foram criadas a fim de promover maior proteção e segurança contra as violências domésticas e o uso de castigos corporais, de tratamento cruel ou degradante contra criança e adolescente (SOUTO, 2018). A partir desse contexto, a violência contra crianças e adolescentes ganhou maior visibilidade, e os castigos corporais, por exemplo, que antes eram "justificados" como forma de educação, passam a ser tipificados como violência, a qual precisa ser notificada, obrigatoriamente, e dirigida aos Conselhos Tutelares e autoridades competentes (SOUTO, 2018).

Dado o estudo-piloto feito nas bases de dados Literatura Latino-americana e do Caribe em Ciências da Saúde (LILACS), Scientific Electronic Library Online (Scielo) e PubMed com os descritores: "Brasil"; "epidemiologia"; "violência" e "criança" na língua portuguesa e na língua inglesa: "Brazil", "epidemiology", "violence" e "children", constataram-se pouquíssimos resultados que abordam a epidemiologia da violência contra crianças e adolescentes.
Portanto, apesar dos investimentos no enfrentamento da violência, por parte do Ministério da Saúde, ainda existem desafios no contexto da divulgação da epidemiologia da violência brasileira. Esses desafios se intensificam ainda mais quando a pesquisa se restringe ao Estado de Minas Gerais, pois os resultados são menores. Por isso, o presente estudo se faz importante, buscando assim ampliar a visibilidade sobre essa casuística e para que o Poder Público, os profissionais da saúde, os assistentes sociais e outros profissionais, que trabalham no atendimento dessa faixa etária possam entender melhor a epidemiologia e, capacitar-se na área e reconhecer e atender às vítimas.

Dessa forma, o presente estudo tem o objetivo de descrever o perfil epidemiológico dos casos de violência praticados contra a população infantil de áreas urbanas do Estado de Minas Gerais.

\section{Material e Métodos}

Realizou-se um estudo descritivo e documental, entre o período de janeiro de 2007 a dezembro de 2017, a partir de dados secundários obtidos no Sistema de Informação de Agravos de Notificação (SINAN) do Estado de Minas Gerais.

Minas Gerais é o quarto maior Estado brasileiro, com uma

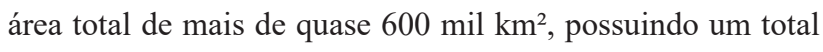
de 853 municípios. O Estado está localizado na região Sudeste e conta com uma população residente estimada de 21.119.536 habitantes, sendo que destes, 5.977.485 têm 19 anos ou menos, segundo dados do Instituto Brasileiro de Geografia e Estatística (IBGE) para o ano de 2017 (BRASIL, 2019).

Foram incluídos os dados de violência contra crianças (de 0 a 18 anos) registrados no SINAN, no período de janeiro de 2007 a dezembro de 2017, do Estado de Minas Gerais. A faixa etária do estudo presente foi baseada de acordo com a Organização Mundial da Saúde (1986), que considera criança até os 19 anos. Foram analisadas as seguintes variáveis referentes à vítima: número de casos e óbitos por violência, faixa etária, raça, cor, sexo, escolaridade, zona de ocorrência e situação conjugal.

Em relação ao ato de violência foram avaliados os tipos de violências (financeira/econômica, negligência/abandono, trabalho infantil, física, psicológica/moral, tortura, sexual e tráfico de seres humanos) e as consequências do ato (gravidez, doença sexualmente transmissíveis, tentativa de suicídio, transtorno mental, tratamento comportamental, estresse pós traumático e outras consequências). Do agressor foram analisados sexo, a relação com a pessoa atendida ou grau de parentesco e se fez uso de álcool.

Para a tabulação dos dados oriundos da plataforma SINAN, foi utilizado o programa Tabwin 3.2, fornecido pelo Ministério da Saúde, sendo também utilizado o programa Microsoft Office Excel 2016 para a construção dos gráficos e tabelas. Em seguida, os dados passaram por uma análise estatística descritiva utilizando-se medidas de frequência. 
Os coeficientes de incidência dos casos de violência contra a criança foram calculados de acordo com o município de ocorrência em Minas Gerais e por ano do estudo apresentados com base nos casos notificados (calculados por 100 mil habitantes). Para análise da incidência foram utilizados dados das estimativas populacionais obtidas a partir dos censos demográficos do Instituto Brasileiro de Geografia e Estatística (IBGE). Além disso, foi utilizada a média aritmética dos respectivos coeficientes anuais para os cálculos dos coeficientes médios de incidência.

Os dados analisados foram extraídos da plataforma de dados secundários do Ministério da Saúde, não nominal, a qual todos os cidadãos brasileiros gozam de livre acesso. Sendo assim, não se faz necessário a emissão de parecer de um Comitê de Ética em Pesquisa (CEP). Os aspectos éticos legais foram respeitados.

\section{Resultados e Discussão}

Diante da análise dos dados executada, foi possível perceber que ocorreram 693.495 notificações de casos de violência contra a criança e ao adolescente no Estado de Minas Gerais, dentro do intervalo de tempo de 2007 até 2017. Nota-se, então, uma incidência média de 3,28 casos a cada mil habitantes.

Dessa análise, de acordo com o gráfico da Figura 1, é possível perceber um crescimento de 347,4\% no ano de 2008 em relação aos casos notificados em $2007 ; 1.182,3 \%$ para o ano de 2009, seguido de crescimento exponencial em toda a série analisada.

Figura 1 - Número de casos por ano de violência contra a criança notificados no Sistema de Informação de Agravos de Notificação de 2007 a 2017

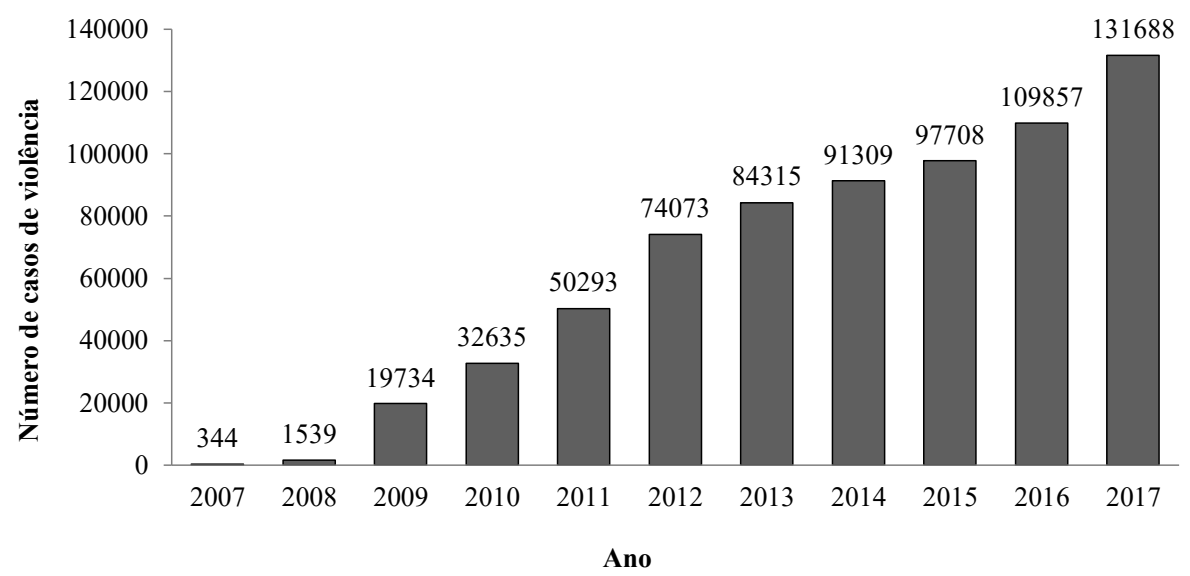

Fonte: Sistema de Informação de Agravos de Notificação.

Do total de notificações feitas, 166.500 (24,0\%) são de crianças entre zero a quatro anos (Quadro 1), sendo que entre esses indivíduos, 9,4\% são menores de um ano. As notificações de crianças de cinco a nove anos assumem a menor frequência, com $97.795(14,1 \%)$ e a faixa com mais notificações, de 15 a 19 anos, sendo representada por 256.612 (37,0\%) casos, sendo dentre esse porcentual o ápice de casos notificados aos 17 anos, com frequência de 16,7\%.

Quadro 1 - Perfil das vítimas de violência, segundo as variáveis, idade da vítima e zona de ocorrência da violência, de acordo com os critérios de evolução da agressão, notificadas no Sistema de Informação de Agravos de Notificação de 2007 a 2017

\begin{tabular}{|l|c|c|c|c|}
\hline \multicolumn{1}{|c|}{ Idade } & Número de Casos & Frequência (\%) & Número de Óbitos & Frequência (\%) \\
\hline 0 a 4 anos & 166500 & 24,0 & 585 & 16,3 \\
\hline 5 a 9 anos & 97795 & 14,1 & 161 & 4,5 \\
\hline 10 a 14 anos & 172578 & 24,9 & 447 & 12,4 \\
\hline 15 a 19 anos & 256612 & 37,0 & 2407 & 66,9 \\
\hline Zona da ocorrência & & & & \\
\hline Urbana & 529.353 & 76,33 & 2.458 & 68,28 \\
\hline Rural & 48.577 & 7,00 & 354 & 9,53 \\
\hline Periurbana & 6.280 & 0,91 & 65 & 1,80 \\
\hline Ignorado ou branco & 109.285 & 15,76 & 734 & 20,39 \\
\hline Total & 693485 & 100 & 3600 & \\
\hline
\end{tabular}

Fonte: Sistema de Informação de Agravos de Notificação.

Os números de óbitos, dos casos notificados, são de 161 óbitos $(4,5 \%)$ de cinco a nove anos na faixa etária que detém o menor índice, seguindo o menor índice de notificações.
Enquanto a faixa de 15 a 19 anos é a que detém maior número de óbitos com 2.407 óbitos (66,9\%), também seguindo o maior índice de notificações. 
Além disso, os dados mostram que dos 693.485 (0,51\%) casos totais de violência notificados resultam em óbitos, isto é, 3.600 óbitos por violência notificados. Esse percentual de óbitos se mostra mais expressivo ao se analisar a faixa etária específica dos 15 aos 19 anos, mencionada anteriormente, na qual a taxa sobe para $0,93 \%$, visto que para 256.612 casos de violência notificados há 2.407 óbitos.

As vítimas (Quadro 2) foram predominantemente negras (pretos e pardos) $304.296(43,88 \%)$ do sexo feminino 421.715 (60,81\%). A maioria das vítimas não tinha o Ensino Fundamental completo 196.914 (28,39\%). A zona predominante de ocorrência da violência foi a urbana 529.353 (76,33\%) (Quadro 1) e o estado civil, mais frequente das vítimas, foi solteiro $291.259(42,00 \%)$.

Quadro 2 - Caracterização da vítima a partir de escolaridade, de raça, de sexo, zona de residência e situação conjugal notificada no Sistema de Informação de Agravos de Notificação de 2007 a 2017

\begin{tabular}{|l|c|c|}
\hline Caracterização da Vítima & $\begin{array}{c}\text { Número } \\
\text { de Casos }\end{array}$ & $\begin{array}{c}\text { Frequência } \\
\text { (\%) }\end{array}$ \\
\hline Escolaridade & & \\
\hline Analfabeto & 2506 & 0,4 \\
\hline Ensino Fundamental incompleto & 196914 & 28,4 \\
\hline Ensino Fundamental completo & 84235 & 12,2 \\
\hline Ensino Médio completo & 23512 & 3,4 \\
\hline Educação Superior completa & 515 & 0,1 \\
\hline Não se aplica & 206702 & 29,8 \\
\hline Ignorado/Branco & 179111 & 25,8 \\
\hline Raça & \multicolumn{2}{|c|}{} \\
\hline Branca & 255589 & 36,9 \\
\hline Preta & 48361 & 7,0 \\
\hline Amarela & 4148 & 0,6 \\
\hline Parda & 255935 & 36,9 \\
\hline Indígena & 5818 & 0,8 \\
\hline Ignorado/Branco & 123644 & 17,8 \\
\hline Sexo & \multicolumn{2}{|c|}{} \\
\hline Masculino & 271129 & 39,1 \\
\hline Feminino & 421715 & 60,8 \\
\hline Ignorado & 651 & 0,1 \\
\hline Situação conjugal & 291259 & 42,0 \\
\hline Solteiro & 28530 & 4,1 \\
\hline Casado/União consensual & 2415 & 0,3 \\
\hline Separado/Viúvo & 84828 & 12,2 \\
\hline Não se aplica & & \\
\hline Ignorado/Branco & & \\
\hline Fonte: Sistema de Informação de Agravos de Notificação. \\
\hline
\end{tabular}

Ao observar os dados referentes à "Caracterização do tipo de violência" (Quadro 3), o tipo que ocupa o primeiro lugar é a "violência física", com 332.369 (47,93\%) casos notificados. Entre os óbitos, também presentes no Quadro 3, por tipo de violência, por "física" ocupa primeiro lugar com 3.053 casos e por "negligência/abandono" em segundo lugar com 359 casos, acompanhando o primeiro e segundo lugar de número de casos de violência. No entanto, o tipo que ocupa o terceiro lugar em óbitos é violência "psicológica" com 227 mortes, que não coincide com o terceiro lugar em números de casos, que foi "sexual" e em óbitos que ocupou a quinta posição.
Quadro 3 - Caracterização do tipo de violência sofrida/infligida notificada no Sistema de Informação de Agravos de Notificação de 2007 a 2017

\begin{tabular}{|c|c|c|c|c|}
\hline $\begin{array}{c}\text { Caracterização } \\
\text { do tipo de } \\
\text { Violência }\end{array}$ & Casos & $\begin{array}{c}\text { Frequência } \\
(\%)\end{array}$ & Óbitos & $\begin{array}{c}\text { Frequência } \\
(\%)\end{array}$ \\
\hline \multicolumn{5}{|c|}{ Financeira/Econômica } \\
\hline Sim & 4032 & 0,58 & 5 & 0,14 \\
\hline Não & 602887 & 86,93 & 2866 & 79,61 \\
\hline Ignorado/Branco & 86576 & 12,48 & 729 & 20,25 \\
\hline \multicolumn{5}{|c|}{ Negligência/Abandono } \\
\hline $\mathrm{Sim}$ & 169078 & 24,38 & 359 & 9,97 \\
\hline Não & 450437 & 64,95 & 2564 & 71,22 \\
\hline Ignorado/Branco & 73980 & 10,67 & 677 & 18,81 \\
\hline \multicolumn{5}{|l|}{ Trabalho Infantil } \\
\hline Sim & 4565 & 0,66 & 7 & 0,19 \\
\hline Não & 602560 & 86,89 & 2871 & 79,75 \\
\hline Ignorado/Branco & 86370 & 12,45 & 722 & 20,06 \\
\hline \multicolumn{5}{|l|}{ Física } \\
\hline $\mathrm{Sim}$ & 332369 & 47,93 & 3053 & 84,78 \\
\hline Não & 304194 & 43,86 & 415 & 11,52 \\
\hline Ignorado/Branco & 56932 & 8,21 & 132 & 3,67 \\
\hline \multicolumn{5}{|c|}{ Psicológica/Moral } \\
\hline Sim & 137857 & 19,88 & 227 & 6,31 \\
\hline Não & 471755 & 68,03 & 2635 & 73,19 \\
\hline Ignorado/Branco & 83883 & 12,10 & 738 & 20,50 \\
\hline \multicolumn{5}{|l|}{ Tortura } \\
\hline Sim & 14673 & 2,12 & 130 & 3,61 \\
\hline Não & 589122 & 84,95 & 2721 & 75,58 \\
\hline Ignorado/Branco & 89700 & 12,93 & 749 & 20,81 \\
\hline \multicolumn{5}{|l|}{ Sexual } \\
\hline Sim & 165706 & 23,89 & 114 & 3,17 \\
\hline Não & 451368 & 65,09 & 2763 & 76,75 \\
\hline Ignorado/Branco & 76421 & 11,02 & 723 & 20,08 \\
\hline \multicolumn{5}{|c|}{ Tráfico de Seres Humanos } \\
\hline Sim & 446 & 0,06 & 3 & 0,08 \\
\hline Não & 607308 & 87,57 & 2882 & 80,06 \\
\hline Ignorado/Branco & 85741 & 12,36 & 715 & 19,86 \\
\hline
\end{tabular}

Quando são analisadas as "consequências das violências" (Quadro 4), evidencia-se 25.237 casos (3,64\%) de "estresse pós-traumático", sendo essa a principal consequência entre as notificadas deste estudo. Ainda, entre os casos, observa-se o total de 15.264 casos $(2,20 \%)$ de "transtornos comportamentais", sendo essa a segunda consequência com mais casos. Outra consequência a ser apontada é a "tentativa de suicídio", presente em 1,41\% dos casos registrados.

Quadro 4 - Consequências dos episódios violentos notificadas no Sistema de Informação de Agravos de Notificação de 2007 a 2017

\begin{tabular}{|c|c|c|}
\hline $\begin{array}{c}\text { Consequências da } \\
\text { Violência }\end{array}$ & Número de casos & Frequência $(\%)$ \\
\hline \multicolumn{3}{|l|}{ Gravidez } \\
\hline $\mathrm{Sim}$ & 7027 & 1,01 \\
\hline Não & 64072 & 9,24 \\
\hline Não se aplica & 256232 & 36,95 \\
\hline Ignorado/Branco & 366164 & 52,8 \\
\hline \multicolumn{3}{|c|}{ Doenças Sexualmente Transmissíveis } \\
\hline Sim & 2879 & 0,42 \\
\hline
\end{tabular}




\begin{tabular}{|c|c|c|}
\hline \begin{tabular}{|c|}
$\begin{array}{c}\text { Consequências da } \\
\text { Violência }\end{array}$ \\
\end{tabular} & Número de casos & Frequência $(\%)$ \\
\hline Não & 145614 & 21 \\
\hline Não se aplica & 94068 & 13,56 \\
\hline Ignorado/Branco & 450932 & 65,02 \\
\hline \multicolumn{3}{|l|}{ Tentativa de Suicídio } \\
\hline Sim & 9755 & 1,41 \\
\hline Não & 153498 & 22,13 \\
\hline Não se aplica & 87975 & 12,69 \\
\hline Ignorado/Branco & 442267 & 63,77 \\
\hline \multicolumn{3}{|l|}{ Transtorno Mental } \\
\hline Sim & 2468 & 0,36 \\
\hline Não & 158213 & 22,81 \\
\hline Não se aplica & 79539 & 11,47 \\
\hline Ignorado/Branco & 453275 & 65,36 \\
\hline \multicolumn{3}{|c|}{ Transtorno Comportamental } \\
\hline Sim & 15264 & 2,20 \\
\hline Não & 146505 & 21,13 \\
\hline Não se aplica & 78445 & 11,31 \\
\hline Ignorado/Branco & 453281 & 65,36 \\
\hline \multicolumn{3}{|c|}{ Estresse Pós-Traumático } \\
\hline Sim & 25237 & 3,64 \\
\hline Não & 137321 & 19,80 \\
\hline Não se aplica & 77086 & 11,12 \\
\hline Ignorado/Branco & 453851 & 65,44 \\
\hline \multicolumn{3}{|c|}{ Outras Consequências } \\
\hline $\mathrm{Sim}$ & 7738 & 1,12 \\
\hline Não & 150014 & 21,63 \\
\hline Não se aplica & 75765 & 10,93 \\
\hline Ignorado/Branco & 459978 & 66,33 \\
\hline
\end{tabular}

Fonte: Sistema de Informação de Agravos de Notificação.

Sobre o perfil dos agressores presentes nas notificações, em 330.046 casos (47,59\%) eram do sexo masculino, em 159.609 $(23,02 \%)$ eram do sexo feminino e em 75.427 (10,88\%). Sobre o grau de parentesco, em 112.669 (16,25\%) dos casos a agressão foi cometida pelo pai, em 156.188 (22,52\%) foi cometida pela mãe, em 101.886 (14,69\%) cometida por amigos ou conhecidos e, em $70.821(10,21 \%)$ cometida por desconhecidos. Além disso, em 98.563 (14,21\%) a agressão foi precedida pelo uso de álcool.

Os dados coletados neste estudo ressaltam alguns pontos que merecem atenção e discussão, a fim de se entender o cenário da violência mostrado pelos resultados contra crianças e adolescentes no Estado de Minas Gerais. Para tal realização, partiu-se da perspectiva de que a violência contra crianças surge em fatos políticos, econômicos e culturais, que são traduzidos nas relações cotidianas interpessoais (ROCHA, 2018).

A partir da análise realizada, é notório que o número de notificações de violência contra crianças nos últimos anos têm aumentado consideravelmente. Além disso, o tipo da violência tem se apresentado multifatorial, perpassando pela violência física, sexual, por negligência ou abandono, violência psicológica ou moral, entre outros tipos, que variam de acordo com os contextos socioculturais e econômicos
(SILVA; MELO, 2018).

A análise dos dados levantados pelo SINAN do Estado de Minas Gerais, no período de tempo entre 2007 e 2017, que demonstra que desde o ano de 2007 o número de casos notificados apresenta um crescimento importante, sendo que os números de casos registrados aumentaram entre 2007 até $2009 \mathrm{em}$ taxas percentuais anuais maiores e, do ano de 2010 ao ano de 2015, embora tenham apresentado um decréscimo no crescimento anual percentual, o número absoluto de casos seguiu crescendo, tendo o seu crescimento relativo novamente aumentado do ano de 2016 até 2017, atingindo maior número no ano de 2017.

Em relação ao aumento das notificações, nos últimos cinco anos registrados, também é muito importante ressaltar que essa configuração analisada pode não revelar, por si só, um aumento da violência no Estado, mas um aumento do número de notificações de profissionais, o que pode ter correlação com o fato de ter se tornado um agravo de notificação compulsória. É válido também destacar o conhecimento do ECA, por parte dos profissionais de saúde, quanto à identificação e notificação de casos de violência, favorecendo o preenchimento da Ficha de Notificação Individual do SINAN (BRASIL, 1990).

Há autores que apontam que o número de notificações e denúncias no Brasil não são fidedignos em função de alguns obstáculos existentes na sociedade brasileira. Exemplos disso são falta de articulação e de comunicação entre a saúde e outros setores da sociedade; a ausência de uma rede que ofereça um maior e melhor suporte; a capacitação dos profissionais para identificação e notificação dos casos de uma forma mais rápida; a pouca importância que é dada ao ato de identificação e de notificação dos casos de violência contra crianças; e a dificuldade de atuação dos mecanismos sociais de proteção à criança, assim como o pouco apoio institucional para a realização das notificações. Tudo isso pode contribuir para um número de notificações inferior ao número de casos reais presentes na sociedade (BEDO; LOURENÇO, 2019).

Para além das parcerias e propostas de intervenção comunitárias, o enfrentamento da violência infantil necessita da criação e do fortalecimento de redes de atendimento e de suportes, além de intervenções comunitárias para trabalhar valores como solidariedade e a cooperação entre as pessoas. Nesse sentido, tais valores comunitários são apontados enquanto o eixo central, que fundamenta as intervenções para melhorar o sistema de notificações e gerar dados fidedignos (BEDO; LOURENÇO, 2019). É preciso que exista, por parte do profissional, uma maior reflexão quanto ao seu papel diante da realidade da violência, o que possibilita uma maior compreensão da importância de trabalhar de forma mais ampliada (SILVA; MELO, 2018).

Ainda, sobre os resultados, ao analisar a idade das crianças e adolescentes vítimas de violência, nos casos estudados, a faixa etária com maior número de notificações é a de 15 a 19 anos, com 256.612 casos notificados, dentro dessa, a idade 
com maior frequência é a de 17 anos. No entanto, estes dados de Minas Gerais divergem dos dados mundiais da Organização Mundial da Saúde, que afirma que a faixa etária mais afetada pela violência é de cinco a nove anos, seguida das crianças de 10 a 14 anos de idade (WHO, 2002).

É perceptível que, dadas às características das vítimas, que foram informadas pelo SINAN, embora apresentem diferentes graus de instrução, o que se destaca perante os demais é o "Ensino Fundamental incompleto", caracterizando um predomínio de baixa escolaridade das vítimas. Este pode ser visto como um fator importante, em que a falta do acesso à informação coincide com o perfil do alvo da violência.

Ainda, sobre as vítimas, o aspecto étnico interfere, mostrando que crianças negras (pretas e pardas) sofrem mais com a violência doméstica que as brancas no Estado de Minas Gerais. Esse dado vai ao encontro de outros estudos que mostram que, historicamente, as crianças negras são alvos de violência e permanecem sendo, no cenário atual, tanto pelas condições de trabalho que exercem quanto por preconceitos e práticas racistas enraizados na cultura dominante (CAMARGO; ALVES; QUIRINO, 2005).

Sobre a zona de residência das vítimas, os casos se expressam mais em vítimas de áreas urbanas, que pode ser um resultado de uma presença mais intensa de sistemas de saúde notificadores, nesses ambientes, que na área rural. Esse dado dialoga com estudos que apontam contrastes entre o acesso à saúde nas diferentes regiões de um município, que minimiza a busca de auxílio para a saúde em moradores de áreas rurais (ARRUDA et al., 2018).

Entre os episódios de violência das notificações, o tipo de violência que apresenta um maior índice de ocorrência é a violência física, sucedido por negligência e abandono. Esse resultado pode não ser de fato o que ocorre na prática, mas ocupa as modalidades mais notificadas em função de consequências visíveis aos profissionais. Já as violências "psicológica/moral”, como são de difícil identificação, são menos notificadas, o que não significa, necessariamente, que ocorrem em menor intensidade (QUADROS et al. 2016).

Todos esses tipos de violência causam consequências, como mostram os dados apresentados na Tabela 5, com altos índices de "estresse pós-traumático", seguido de "transtorno comportamental" e de "tentativa de suicídio", consequências agravadas por tais dados se referirem às crianças, indivíduos que estão no início de suas vidas. Uma vez que, qualquer violência cometida às crianças é capaz de gerar consequências graves em seu desenvolvimento e em sua formação como indivíduo e cidadão, podendo apresentar tanto mudanças em seu comportamento como distúrbios de conduta (REIS et al., 2018). Além disso, é importante salientar que informações que foram consideradas como "ignoradas ou em branco", apresentam-se como maioria para todas variáveis analisadas de consequências, dificultando mensurar as reais causas da violência, que podem ser ainda maiores.

Sobre a mãe ser o parente mais frequente a agredir as vítimas, o resultado pode também ser comparado a outros estudos, em que as mães são, na maior parte das vezes, as principais agressoras por passarem mais tempo que o pai cuidando dos filhos e acompanhá-los em atendimentos médicos, que geram as notificações (SOUTO, 2018).

Perante os dados levantados, desde o aumento de casos notificados até as consequências deles para as crianças, faz-se necessárias ações de políticas públicas eficazes para amenizar todo e qualquer tipo de violência sofrida. Além disso, é importante que os profissionais da área da saúde estejam aptos e capacitados para notificar e saber intervir em casos de violência contra as crianças, adotando postura e estratégias de promoção e de prevenção de saúde a esse tipo de população. É preciso, por parte dos profissionais e da sociedade, a prática do que o Estatuto da Criança e do Adolescente (ECA) preconiza (BRASIL, 1990), como direito à vida, à saúde e tantos outros aspectos que são de suma importância no desenvolvimento saudável de qualquer criança.

\section{Conclusão}

Os dados do presente estudo mostraram que as vítimas foram, predominantemente, do sexo feminino, negras, na faixa etária de 15 a 19 anos, com baixa escolaridade. Observou-se, ainda, predomínio da violência física, praticada por familiar, com relato de uso de álcool. Conclui-se, portanto, que esse perfil supracitado é o mais vulnerável, de acordo com essa pesquisa, para sofrer violências. Assim, políticas públicas para minimizar essa problemática são fundamentais, buscando se evitar que os mesmos jovens continuem sendo violentados e novos jovens venham a se tornar vítimas da violência.

Além disso, diante da subnotificação, no sistema de saúde brasileiro, somada ao fato de que toda faixa etária pesquisada neste trabalho sofreu violências das mais diversas formas, essa conclusão não pode desconsiderar que as ações públicas devem abranger toda criança e adolescente. Com isso, mesmo que traçado um perfil epidemiológico com destaque para determinadas formas de violência e padrões de vítimas, o combate deve ser amplo. Conclui-se, assim, que as crianças e adolescentes no Estado de Minas Gerais necessitam de maior atenção no que diz respeito à prevenção e à promoção à saúde e de estabelecimento de ações efetivas no combate à violência.

\section{Referências}

ARRUDA, N.M.; MAIA, A.G.; ALVES, L.C. Desigualdade no acesso à saúde entre as áreas urbanas e rurais do Brasil: uma decomposição de fatores entre 1998 a 2008. Cad. Saúde Pública, v.34, 2018. doi: 10.1590/0102-311X00213816

BRASIL. Presidência da República. Casa Civil. Subchefia para Assuntos Jurídicos. Lei n. ${ }^{\circ}$ 8.069, de 13 de julho de 1990.

BRASIL. IBGE - Instituto Brasileiro de Geografia e Estatística. Brasil em Síntese. Brasília: IBGE, 2019.

BRASIL. Presidência da República. Lei no 8.069, de 13 de julho de 1990 .

BEDO, L.C.; LOURENÇO, E.D. O processo de notificação da 
violência na política de saúde e as contribuições do serviço social para o debate. Congresso Brasileiro de Assistentes Sociais 2019, v. 16, n. 1, 2019.

CAMARGO, C.L.de; ALVES, E.S.; QUIRINO, M.D.; Violência contra crianças e adolescentes negros: uma abordagem histórica. Texto Contexto Enferm., v.14, n.4, p.608-615, 2005. doi: 10.1590/ S0104-07072005000400019.

COSTA, M.C.O. et al. O perfil da violência contra crianças e adolescentes, segundo registros de Conselhos Tutelares: vítimas, agressores e manifestações de violência. Ciênc. Saúde Coletiva, v.12, p.1129-1141, 2007. doi: 10.1590/S141381232007000500010

KLIPPEL, Y; CAMARGO, D. Processo participativo entre profissionais de saúde para integrar o atendimento à criança vítima de violência. Rev. Pesq. Práticas Psicossoc., v.10, n.2, p.340-353, 2015.

LOBATO, G.R.; MORAES, C.L.; NASCIMENTO, M.C. do. Desafios da atenção à violência doméstica contra crianças e adolescentes no Programa Saúde da Família em cidade de médio porte do Estado do Rio de Janeiro, Brasil. Cad. Saúde Pública, v.28, p.1749-1758, 2012. doi: 10.1590/S0102-311X2012000900013.

LONGO, I. S. To be adolescent and child in the Brazilian society: passed and present of the history of youthful rights. In: CONGRESSO INTERNACIONAL DE PEDAGOGIA SOCIAL, 3, São Paulo. 2010.

MACEDO, D. M. et al. Revisão sistemática de estudos sobre registro de violência contra crianças e adolescentes no Brasil. Ciênc. Saúde Coletiva, v.24, n.2, p.487-496, 2019. doi: 10.1590/1413-81232018242.34132016.

MAGALHÃES, J. R. F. D. et al. Violência contra crianças e adolescentes negros: uma abordagem histórica. Texto ContextoEnferm., v.14, n.4, p.608-615, 2005. doi: 10.5935/14148145.20170003.

QUADROS, M. N. D. et al. Situação da violência contra crianças e adolescentes no Brasil. Enferm. Global, v. 44, p. 174-85, 2016.

REIS, D.M; PRATA, L.C.G; PARRA, C.R. O impacto da violência intrafamiliar no desenvolvimento psíquico infantil. Psicologia.pt, 2018.

ROCHA, R. D. C. et al. Violência velada e revelada contra idosos em Minas Gerais-Brasil: análise de denúncias e notificações. Revista Saúde em Debate. Dez. pp. 81-94, 2018. doi: 10.1590/0103-11042018s406.

SILVA, J. C. T.; MELO, S. C. de A.; Violência Infantil: atuação do psicólogo no processo de auxílio à criança. Psicol. Saúde Debate, v.4, n.1, p.61-84, 2018. doi: 10.22289/2446-922X.V4N1A4

SOUTO, D.F. et al. Violence against children and adolescents: profile and tendencies resulting from Law 13.010. Rev. Bras. Enferm., v.71, p.1237-1246, 2018. doi: 10.1590/0034-71672017-0048

WHO - World Health Organization. Consolidated Guidelines On: The Use of Antiretroviral Drugs for Treating and Preventing Hiv Infection. Recommendations for a public Health Approach. 2013. Disponível em: https://www.who.int/hiv/pub/guidelines/ arv2013/intro/keyterms/en/

WHO-World Health Organization. Inspire: sete estratégias para pôr fim à violência contra crianças. 2016. Disponível em: https://apps. who.int/iris/bitstream/handle/10665/207717/9789241565356por.pdf?ua=1

WHO - World Health Organization. Relatório mundial sobre violência e saúde. Genebra: WHO; 2002. 